Research Article

\title{
Fractal-Dimension-Based Walking Trajectory Analysis: A Case Study in Museum J. Armand Bombardier
}

\author{
Xueying Han $\mathbb{D}^{1,2}$ Changhong Zhan $\mathbb{D}^{1,2}$ and Guanghao $\mathrm{Li} \mathbb{1}^{1,2}$ \\ ${ }^{1}$ School of Architecture, Harbin Institute of Technology, Harbin 150006, China \\ ${ }^{2}$ Key Laboratory of Cold Region Urban and Rural Human Settlement Environment Science and Technology, \\ Ministry of Industry and Information Technology (Harbin Institute of Technology), Harbin 150006, China
}

Correspondence should be addressed to Guanghao Li; guanghao.li@hit.edu.cn

Received 21 July 2021; Revised 25 August 2021; Accepted 2 September 2021; Published 8 October 2021

Academic Editor: Ma Jianjun

Copyright ( $) 2021$ Xueying Han et al. This is an open access article distributed under the Creative Commons Attribution License, which permits unrestricted use, distribution, and reproduction in any medium, provided the original work is properly cited.

\begin{abstract}
A comprehensive understanding of the randomness, arbitrariness, and complexity of the visitors' behavior and interaction in a museum is important because it is associated with the design. There is still uncertainty about how to characterize the visitors' behavior and interaction. The fractal dimension was used in this study to indicate the geometrical form of the aged's, the families', and the students' walking trajectories. The study results represented that all three sorts of the walking trajectory fractal-dimensiontime curves fluctuated in the early stage. A remarkable exponential converges could then be observed. The mean fractal dimension after the convergence of the aged's, the families', and the students' walking trajectory was nearly 1.8, 1.6, and 1.2, respectively. Furthermore, the behavior characteristics of these three sorts of visitors were quantified and the reasons were speculated and inferred. The comprehensive consideration of fractal geometry can aid in visitors' behavior modeling and museum design.
\end{abstract}

\section{Introduction}

A qualified museum design could evidently improve visitors' experiences. In recent years, the interactive design theory of museums has been substantially developed. The interactive design method considers the visitors' behavior would influence the design of the building and the deployment of exhibits. This design method is commonly based on the visitors' behavior, as well as the interaction among the visitors, the exhibits, and the museum space [1]. With the aid of interaction research, the architect paid more attention to the visitors' feelings and feedback. The visiting experience has been then remarkably enriched and improved [2]. However, the visitors' behavior represents obviously random, arbitrary, and complex. Limited to the theory and technology, the visitors' behavior and the interaction are difficult to be studied and explained comprehensively.

Modeling the visitors' behavior is the basis of interaction research. The visitors' behavior and interaction would influence the design directly. The interactive communication is the key to the museum design [3]. Different theories exist regarding the quantification of behavior and interaction. These theories may be classified according to indicator and study manner into two main categories $[4,5]$.

The first common manner is mainly focusing on the singleton behavior. Using this method, the singleton behavior, for instance, the visitors' behavior, cognition, and perception, could be studied. Based on the quantification of these behaviors, the interactive design theory would be developed. As the capacity of museums to display information to visitors is acutely limited by physical space, these studies are helpful in museum design [6]. The studies provide evidence that multitouch interactive exhibits which enable visitors to explore large scientific data sets and can provide engaging and effective learning opportunities [7]. Besides, several findings emphasize the importance of carefully considering recruitment decisions in museum research and evaluation [8-10]. The feature of these singletons could be considered as the main reference to designing. However, the interactive design based on the singleton behavior could only provide the fundamental properties of space and exhibit location. It is difficult to 
quantify the influence between different elements, which do not improve the design effectively.

The other theory is mainly focusing on multielement interaction. The elements discussed contain the visitors, the building, and the exhibits. Then, the interaction among these elements would influence the design of the building and the deployment of exhibits. This manner has been significantly developed with the aid of digital technology and device, based on which the interaction between the visitors and the exhibits could be obtained effectively. Then, the researchers implement the sociology environmental psychology and numerical simulation, synthetically to analyze the interaction [11]. In this theoretical frame, the method of increasing visitors' experience through greater interaction and engagement has been discussed [12]. Using digital technology, the building unit, materials, and intelligent equipment could be inserted in the interactive museum $[13,14]$. Although the studies on the multielement interaction provide a method to quantify the interaction, its randomness, arbitrariness, and complexity have not been described reasonably.

The random, arbitrary, and complex features of the interaction would influence the walking trajectory and exhibits design directly. As it is difficult to be studied and explained comprehensively, the researchers and architects have to introduce the assumptions and hypothesis intentionally or use a number of smart equipment to compensate for the inadequate model. This restricts the development of the museum design and the interactive theory.

The fractal theory, which provides a general framework for the study of the random, arbitrary, and complex objectives, has been introduced [15-17]. The fractal dimension of the random curves could be obtained conveniently based on the box-counting method [18]. This fractal dimension calculated by applying the box-counting method is named as box-counting dimension (BCD) [19]. Fractal theory and $\mathrm{BCD}$ have been introduced in the domain of museum design and the interactive theory, which could help us to investigate building's visual complexity [20], to discuss the deploy artistic exhibits [21], and to assess the atmospheric pollution impact on the microstructure of marble surfaces [22].

In this study, one classic type of visitors' interaction, i.e., their walking trajectories were analyzed and discussed based on the fractal geometry. Considering a walking trajectory as a two-dimensional random curve, its geometrical characteristic was quantified. Then, the randomness, arbitrariness, and complexity of the visitors could be studied. It is of great necessity to describe and model the visitor behavior and interaction in the museum, which will be of interest to the researchers and architects.

\section{Box-Counting Method for Numerical Objects}

BCD is one of the common types of fractal dimension that can represent the fractal and geometrical properties of objects [23]. Moreover, the BCD has a significant advantage of being easily measured with unknown gradation [24]. Therefore, it is one of the most widely applied methods of determining the fractal dimensions of objects.
As the algorithm represented in Figure 1, the BCD of a numerical objective could be practically estimated from the slope of a series of points plotted in a log-log plot. The $X$ and $Y$ coordinate of each point could be calculated by $\ln N_{\text {box }}$ and $\ln \delta$, respectively, where $N_{\text {box }}$ represents the smallest number of box of side $\delta$ that covers the objective. Usually, the initial $\delta$ is set as a quarter of the length of the objective's side, and the minimum $\delta$ is the pixel unit. Moreover, the plateaus of the log-log plot should be eliminated. Specifically, if several sequential $N_{\text {box }}$ counted by different $\delta$ is the same, only the first element in this group of points would be retained.

\section{Case Study}

3.1. Museum J. Armand Bombardier. The Museum J. Armand Bombardier (J. AB) located in the Montreal suburb is considered compact and tourist-friendly. There are not many partition walls inside. The action data of tourists could be observed readily. As shown in Figure 2, J. AB contained several main areas, three general exhibition halls, a temporary exhibition hall, a multimedia room, a recreation area, a shopping area, and offices.

In this case study, the Idea Studio, one of the three general exhibition halls, was selected and analyzed. As shown in Figure 3, this hall had three entrances. Entrance A locates the closest to the door of the museum. Therefore, most tourists entered by entrance $A$ and exited by entrance A, B, and C. Besides, the brown block shown in Figure 3 represented the exhibits. The tourist in $\mathrm{J}$. AB consisted of the students, the family, and the aged.

3.2. Geometric Characteristic of Walking Trajectory. Three sorts of tourists were observed and studied in this paper, i.e., the student, the family, and the aged. Ten subjects of each sort were discussed. Five cameras and one key mission equipment were applied to observe and record the tourists' walking trajectories. Then, the data recorded by these cameras and equipment were processed in a holographic 3D optical projection system. Finally, the walking trajectories could be plotted according to the output of the holographic 3D optical projection system. More details about the holographic 3D optical projection system could be found in [25].

The fractal dimension was introduced to quantify the geometrical characteristic of the varied tourists' walking trajectories. A walking trajectory could be considered as a location-time curve in a two-dimensional plane. Noting the index set $T$ as the whole visiting time, $D(t)$ represented the box-counting dimension of the tourist's walking trajectory before the moment $t, t \in T$.

Three sorts of walking trajectories and their $D(t)$ were discussed in this case study.

\section{Results and Discussion}

4.1. Walking Trajectory. The walking trajectories of the ten visitors of each sort were plotted as black lines in Figures 4-6, respectively. The pink point in the record represented the places tourists stopped and stayed. The area of the pink point represented the staying time; the larger 


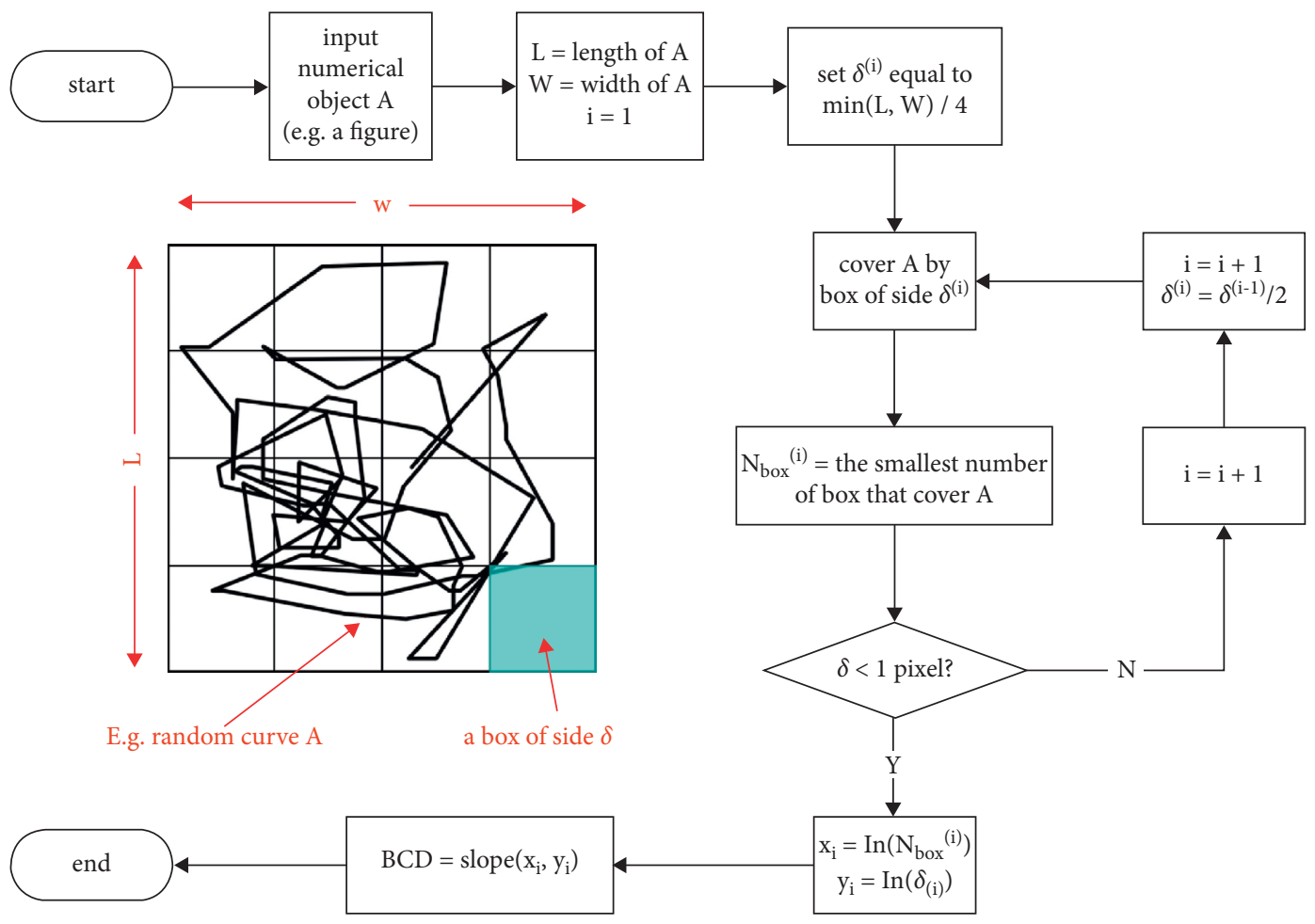

FIGURE 1: Algorithm of the box-counting method.

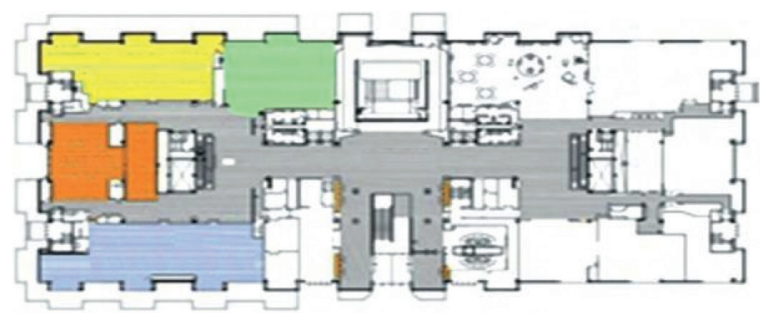

Figure 2: Construction plan of Museum J. Armand Bombardier.

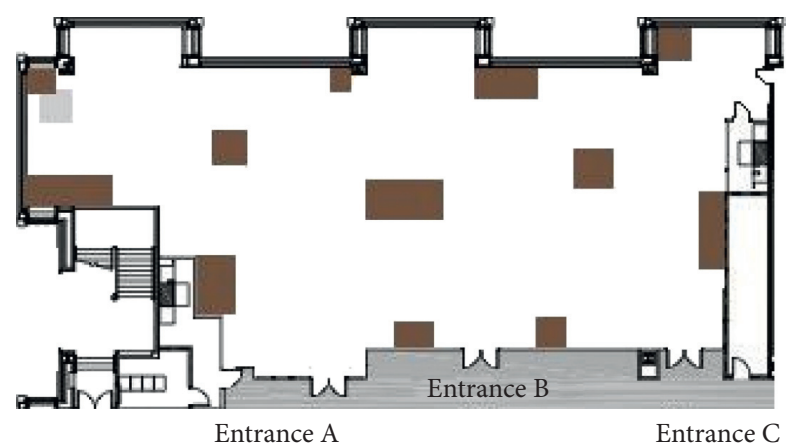

FIgUre 3: Construction plan of the exhibition Idea Studio.

pink area indicated a longer staying time. The minimum staying time in this study was set as 15 seconds. Once the tourist stayed in a location for more than 15 seconds, a pink point would be generated.

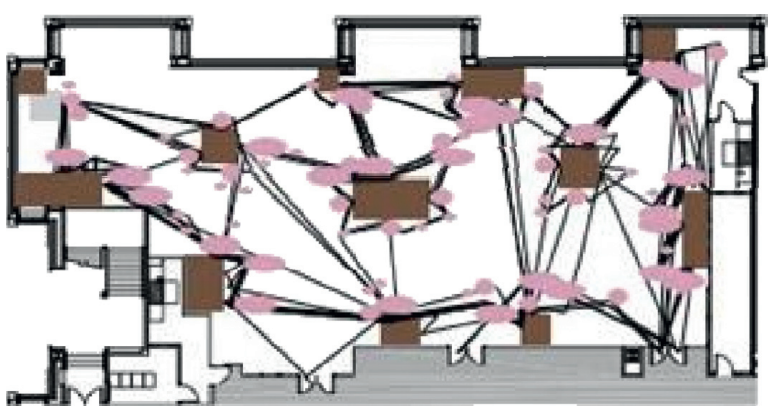

FIgURE 4: Residence status recording of the student.

It should be noticed that the students who visit J. AB usually aim to finish the homework according to the questionnaire survey. They visit the museum in extracurricular time. The walking trajectory shown in Figure 4 demonstrated that the visit route of students is relatively fixed. The students showed not much interest in other unrelated exhibits. In order to finish the homework, the students who visit J. AB preferred to arrive at the specified position, which might be the topic of the homework. Besides, the larger pink points represented that students would stay in this specified position for a longer time. They might spend time finishing the homework closed to the relative exhibit.

The families usually visit J. AB at the weekend. They prefer to follow the route signs marked by the museum. The number of pink points in Figure 5 represented a higher frequency of staying. However, they did not stay for a long time. Meanwhile, according to the walking trajectory shown 


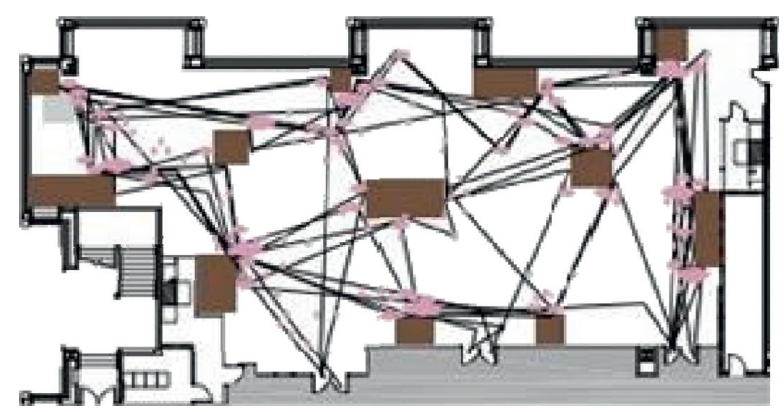

Figure 5: Residence status recording of the family.

in Figure 5, this sort of the visitor did not retrace back too often.

The visiting time of the aged was more arbitrary. The walking trajectory in Figure 6 shows a much more casual visiting route. Besides, the larger pink points represented that the aged would stay near the exhibits for a longer time.

4.2. Box-Counting Dimension of Walking Trajectory. Ten subjects' walking trajectory was randomly selected. Since visitors spent different time periods in $\mathrm{J}$. AB, all the walking time was divided into 1000 parts proportionally. As mentioned in Section 3.2, considering the walking trajectory as a two-dimensional curve, the curve before the moment $t$ could be plotted. Then, the BCD of this part of the subject's walking trajectory could be obtained according to the method introduced in Section 2, which was noted as the BCD of sample 1 before the moment $t$. The average of the BCDs of these ten subjects' walking trajectory could be noted as the BCD before the moment $t$. Finally, a BCD-time curve could be obtained by repeating this operation. This method was drawn schematically in Figure 7. Three sorts of walking trajectories and their $\mathrm{D}(\mathrm{t})$ could be obtained by implementing this method, which is shown in Figure 8.

All the box-counting dimension curves of visitor's walking trajectories were plotted in Figure 8. Before the converge, all the dimensions fluctuated remarkably. Then, the curves converged almost exponentially. After this obvious convergence, all the box-counting dimensions fluctuated slightly in a mean value. The mean fractal dimension of the aged's walking trajectory was the highest. A higher fractal dimension implied a higher complexity. The walking trajectory with a higher fractal dimension usually displays more randomly. The highest fractal dimension might be caused by their arbitrary movement discussed in Section 4.1. Comparatively, the smaller value after converge of the families might indicate that their visiting manners were more regular. The fractal dimension of students' walking trajectory was between the other two sorts. That might be caused by varied homework. Each curve would be discussed as follows.

The box-counting-dimension-time curve plotted in Figure 9 implied that the duration of its fluctuation was the longest. Furthermore, the power function, $y=A x^{B}$, fitted both its upper and lower envelopes well. Two reasons might cause this fluctuation. (1) In its early stages, the geometrical

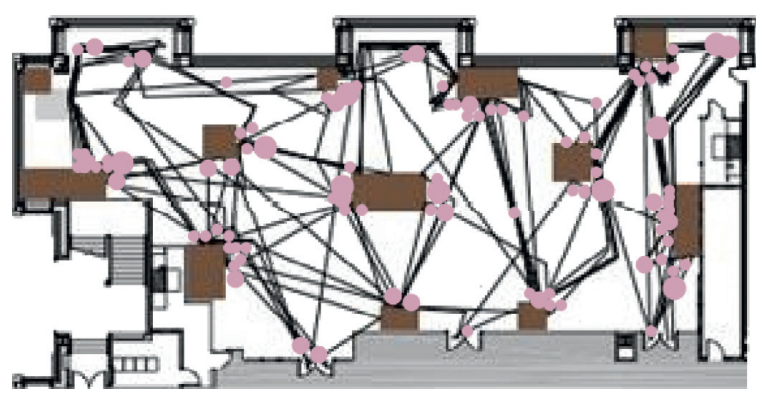

FIGURE 6: Residence status recording of the aged.

and fractal characteristics of the walking trajectory would change with time obviously. $D(t)$ might then be very different from $D(t+\Delta t)$, where $\Delta$ is a small increment of time. (2) In the early stages, the walking trajectory was not that long. The dimensions fluctuated remarkably before the convergence might be caused by the box-counting method introduced in Section 2. Specifically, in the early stages, the walking trajectory was short and more random than the latter. The length and the complexity of the walking trajectories in adjacent moments changed significantly. The number of boxes counted in debut would change remarkably, which caused $D(t)$ fluctuant. Then, the walking trajectory extended with time, the difference between two adjacent moments diminished. Its BCD more and more weakly and finally became stable.

Meanwhile, the mean fractal dimension of the aged's walking trajectory was nearly 1.8. The fractal dimension between 1.0 and 2.0 was commonly seen in the complex and random two-dimensional curves, for instance, the Koch curves (1.2618). This value demonstrated that the aged's walking trajectory is a fractal pattern existing in two-dimensional space.

The box-counting-dimension-time curve plotted in Figure 10 implied that the duration of its fluctuation was shorter than that of the aged. Also, the power function fitted both its upper and lower envelopes well. The coefficients of $A$ and $B$ demonstrated its faster convergency. Meanwhile, the mean fractal dimension of the students' walking trajectory was nearly 1.6. This value demonstrated the aged's walking trajectory more complex and random than the students' walking trajectory.

The box-counting-dimension-time curve plotted in Figure 11 implies that the fluctuation before the convergence was the most slight, and the duration of fluctuation before the convergence of families' BCD was shortest. Also, the power function, $y=A x^{B}$, fitted both its upper and lower envelopes well. Meanwhile, the mean fractal dimension of the students' walking trajectory was nearly 1.2. This value demonstrated the families' visiting path less complex and random than the other two.

The BCD of the students' walking trajectory could be observed hither than the families' walking trajectory. We considered the following two reasons: (a) the BCD-time curves represented the BCD obtained by ten visitors' walking trajectory average. There are three entrances of the museum, students might select a different one. Meanwhile, despite the 


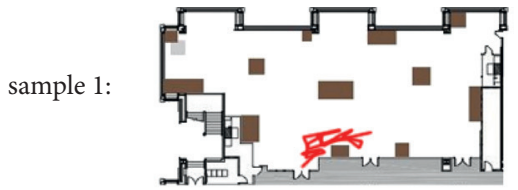

$\mathrm{BCD}(\mathrm{t} 1)$ of sample 1

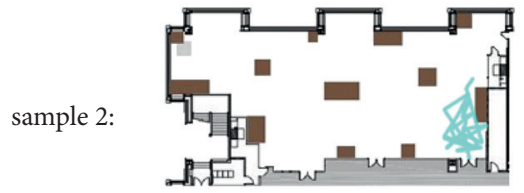

$\mathrm{BCD}(\mathrm{t} 1)$ of sample 2 t2

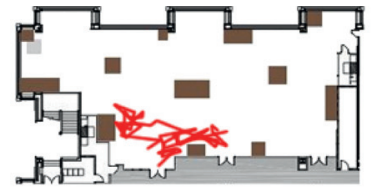

BCD (t2) of sample 1

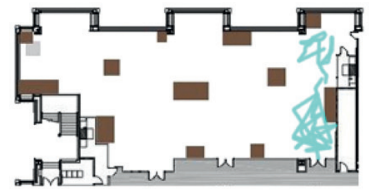

BCD (t1) of sample 2

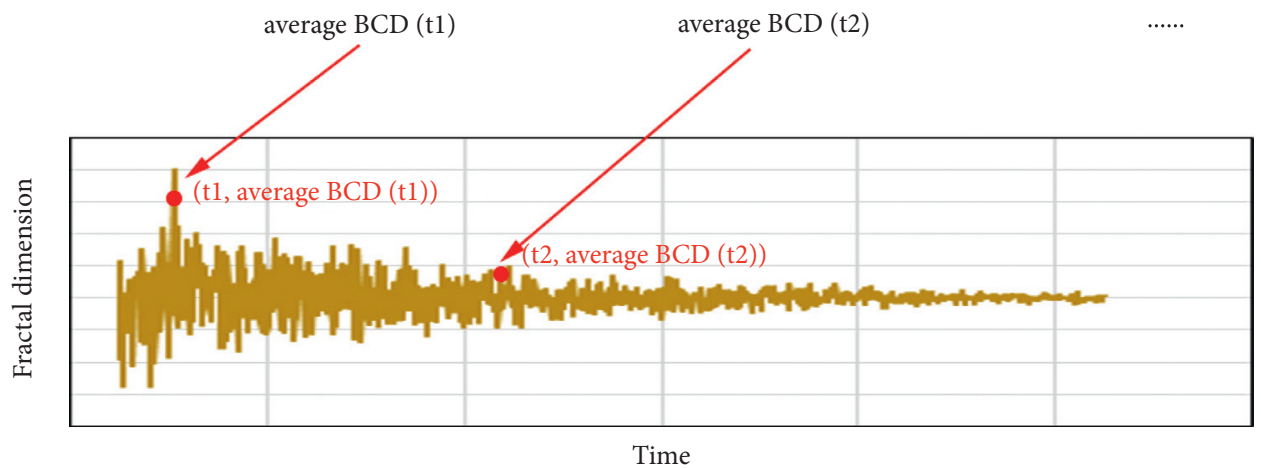

FIgURE 7: Schematic drawn of box-counting-dimension-time curve plotting method.

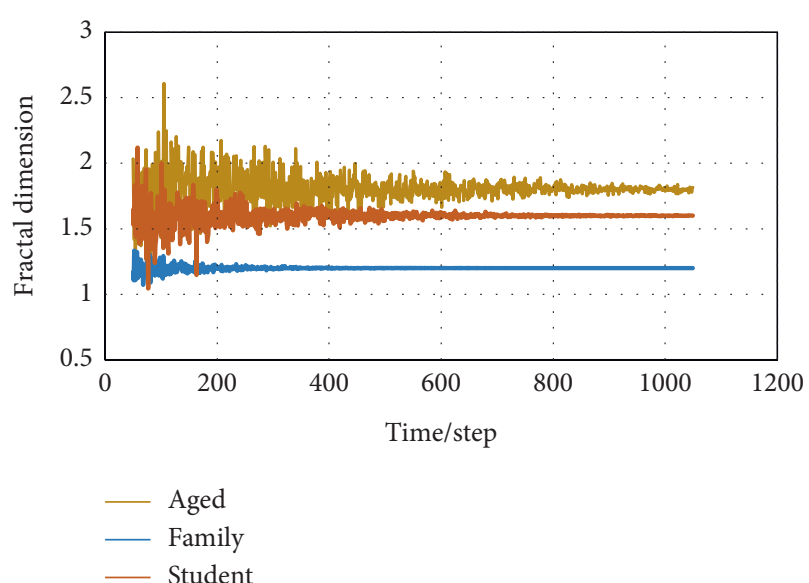

FIGURE 8: Box-counting-dimension-time curve of three sorts of visitors.

same visiting reason of students, i.e., finishing homework, they would work on different exhibits. That is to say, the visiting routes of varied students would differ. (b) Not all the students could arrive at their destination from the entrance directly. They might need to find the way, or get wrong and turn back, or get attracted by other exhibits, and so on. These two reasons might cause their visiting more random and

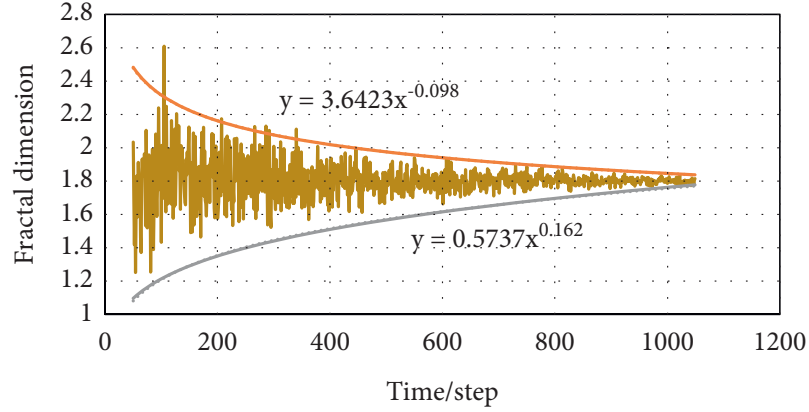

FIGURE 9: Box-counting-dimension-time curve of the aged.

arbitrary. Thus, higher complexity of students' walking trajectory was observed than the families'.

The quantitative discussion above could support the points introduced in Section 4.1. The results and discussion introduced in this study demonstrated that the geometrical characteristic of varied visitors' walking trajectories differed remarkably. The fractal dimension after the converge could quantify this feature. Concerning the geometrical characteristic of varied visitors' walking trajectories as well as their stay time, the museum design could be improved. For instance, in the case of the students for a large proportion of visitors, a wider space as well as several tables and chairs 


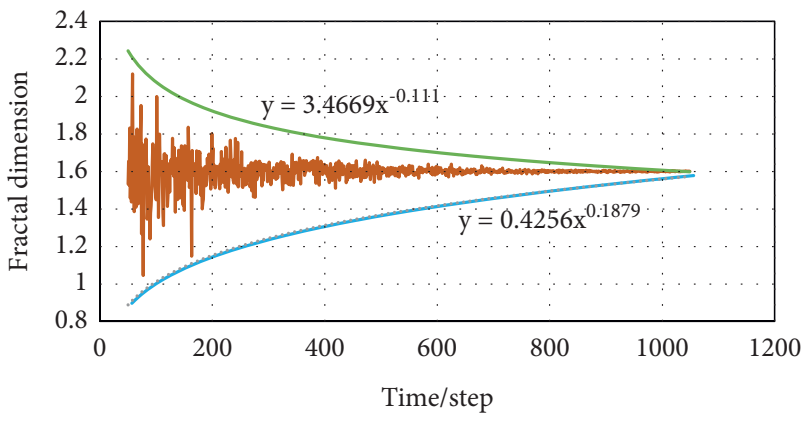

Figure 10: Box-counting-dimension-time curve of students.

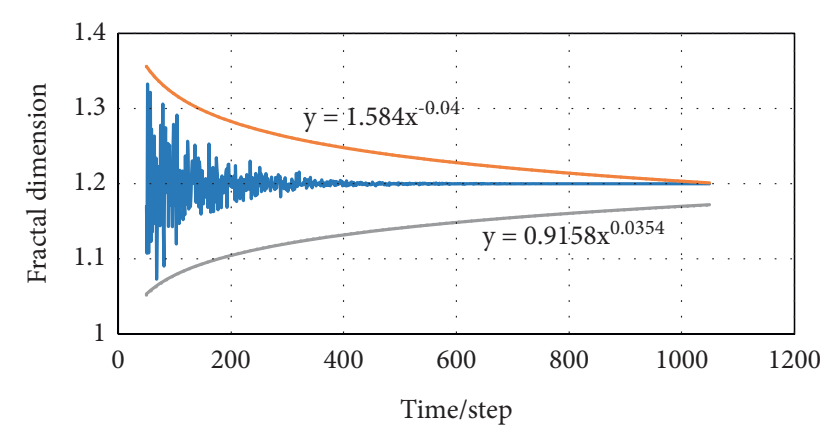

Figure 11: Box-counting-dimension-time curve of families.

would be more preferable in the area of several special exhibit's peripheries. Similar to the students, the higher fractal dimension of the aged's walking trajectories represented that they preferred a casual visit. Fewer partition walls and more bold routing instructions might help these two sorts of visitors. However, comparatively, in order to improve the families' visiting experiences, the designers could add partition walls and exhibits, as well as detailed and interesting descriptions in the museum.

\section{Conclusions}

This study analyzed the geometrical characteristics, the fractal dimension, of varied visitors' walking trajectories in the museum J. AB. Three sorts of visitors, the aged, the family, and the students, were discussed. Their properties were discussed in-depth. The following conclusions can be drawn:

(1) All three sorts of the walking trajectories' fractaldimension-time curves represented a similar pattern. At the early stage, the dimensions fluctuated significantly. A remarkable exponential converges could then be observed. After this convergency, all the dimensions fluctuated slightly in a mean value.

(2) The mean fractal dimension after the convergence of the aged's, the families', and the students' walking trajectory was nearly $1.8,1.6$, and 1.2 , respectively.

(3) The behavior characteristics of these three sorts of visitors were quantified and the reasons were speculated and inferred.
This study contributes to our understanding of characterizing the geometrical form of varied visitors' behavior and its reason. The comprehensive consideration of fractal geometry and can provide a powerful tool for understanding random, arbitrary, and complex interaction. This study could provide some essential information that will help improve efficiency for museum researchers and architects.

\section{Data Availability}

No data were used to support the findings of the study.

\section{Conflicts of Interest}

The authors declare that they have no conflicts of interest.

\section{Acknowledgments}

This work was supported by the Province Heilongjiang Postdoctoral Science Foundation (Grant no. LBH-Z20010).

\section{References}

[1] U. Haque, "Architecture, interaction, systems, AU," Arquitectura y Urbanismo, vol. 149, pp. 1-5, 2006.

[2] Y.-S. Chang, Y.-C. Tsai, and T.-H. Wang, "The user research of interactive app design for 2016 yilan green expo food-ethics museum," in Proceedings of the 2018 IEEE International Conference on Advanced Manufacturing (ICAM), pp. 481484, IEEE, Yunlin, Taiwan, 16-18 November 2018.

[3] C. Kai, "The key of successful museum building is the interaction between construction and exhibition design," Art and Design, vol. 191, no. 3, pp. 12-14, 2009.

[4] M. T. Marshall, N. Dulake, L. Ciolfi, D. Duranti, H. Kockelkorn, and D. Petrelli, "Using tangible smart replicas as controls for an interactive museum exhibition," in Proceedings of the TEI'16: Tenth International Conference on Tangible, Embedded, and Embodied Interaction, pp. 159-167, Eindhoven Netherlands, 14-February-2016.

[5] A. Satyanarayan, R. Russell, J. Hoffswell, and J. Heer, "Reactive vega: a streaming dataflow architecture for declarative interactive visualization," IEEE Transactions on Visualization and Computer Graphics, vol. 22, no. 1, pp. 659-668, 2015.

[6] S. Karaman, A. D. Bagdanov, L. Landucci et al., "Personalized multimedia content delivery on an interactive table by passive observation of museum visitors," Multimedia Tools and Applications, vol. 75, no. 7, pp. 3787-3811, 2016.

[7] M. S. Horn, B. C. Phillips, E. M. Evans, F. Block, J. Diamond, and C. Shen, "Visualizing biological data in museums: visitor learning with an interactive tree of life exhibit," Journal of Research in Science Teaching, vol. 53, no. 6, pp. 895-918, 2016.

[8] S. A. Pattison and T. Shagott, "Participant reactivity in museum research: the effect of cueing visitors at an interactive exhibit," Visitor Studies, vol. 18, no. 2, pp. 214-232, 2015.

[9] A. Agaronov, M. M. Leung, J. M. Garcia et al., "Feasibility and reliability of the system for observing play and leisure activity in youth (SOPLAY) for measuring moderate to vigorous physical activity in children visiting an interactive children's museum exhibition," American Journal of Health Promotion, vol. 32, no. 1, pp. 210-214, 2018.

[10] A. T. Lam, J. Ma, C. Barr et al., "First-hand, immersive fullbody experiences with living cells through interactive 
museum exhibits," Nature Biotechnology, vol. 37, no. 10, pp. 1238-1241, 2019.

[11] J. Pallud, "Impact of interactive technologies on stimulating learning experiences in a museum," Information \& Management, vol. 54, no. 4, pp. 465-478, 2017.

[12] L. Wong, S. Shimojo, Y. Teranishi, T. Yoshihisa, and J. H. Haga, "Interactive museum exhibits with embedded systems: a use-case scenario," Concurrency and Computation: Practice and Experience, vol. 29, no. 13, Article ID e4141, 2017.

[13] K.-K. Kang, J. Lee, C. J. Park, J. W. Kim, and M. H. Lee, "Xtop: an interactive exhibition for building experience with mini-components of stone pagoda," in Proceedings of the 2015 Digital Heritage, vol. 1, pp. 423-424, IEEE, Granada, Spain, October 2015.

[14] L. Ying and T. Shengwwei, "The construction of the building facade building materials-_construction technology and the build facade design interactive teaching reform research," Fujian Architecture \& Construction, vol. 206, no. 8, pp. 104-105, 2015.

[15] T. Wen and K. H. Cheong, "Invited Review: The Fractal Dimension of Complex Networks: A Review," Information Fusion, vol. 73, pp. 87-102, 2021.

[16] X. Wang, B. Yang, L. Jin, Z. Zhang, and X. Xu, "Management and fractal analysis of desiccation cracks of soils with acid contamination," Advances in Civil Engineering, vol. 2020, Article ID 6678620, 9 pages, 2020.

[17] X. Yang, L. Li, H. Dai, and M. Jia, "Effect of fractal dimension in concrete meso-structure on its axial mechanical behavior: a numerical case study," Fractals, vol. 29, no. 2, Article ID 2140011, 2021.

[18] A. Husain, J. Reddy, D. Bisht, and M. Sajid, "Fractal dimension of coastline of Australia," Scientific Reports, vol. 11, no. 1, pp. 1-10, 2021.

[19] E. Rosenberg, "Computing the box counting dimension," in Fractal Dimensions of Networks, pp. 107-129, Springer, Berlin, Germany, 2020.

[20] L. Ma, H. Zhang, and M. Lu, "Building's fractal dimension trend and its application in visual complexity map," Building and Environment, vol. 178, Article ID 106925, 2020.

[21] C.-H. Lan, C.-Y. Hsui, and L.-C. Wei, "A complexity perspective to deploy artistic exhibits," Journal of the Operational Research Society, vol. 56, no. 10, pp. 1151-1158, 2005.

[22] A. Moropoulou, E. Delegou, E. Karaviti, and V. Vlahakis, "Assessment of atmospheric pollution impact on the microstructure of marble surfaces," in Measuring, Monitoring and Modeling Concrete Properties, pp. 695-701, Springer, Berlin, Germany, 2006.

[23] C. Panigrahy, A. Seal, and N. K. Mahato, "Image texture surface analysis using an improved differential box counting based fractal dimension," Powder Technology, vol. 364, pp. 276-299, 2020.

[24] K. Falconer, Fractal Geometry - Mathematical Foundations and Applications, John Wiley \& Sons, Hoboken, NJ, USA, 1990.

[25] T. Nishitsuji, T. Kakue, D. Blinder, T. Shimobaba, and T. Ito, "An interactive holographic projection system that uses a hand-drawn interface with a consumer CPU," Scientific Reports, vol. 11, no. 1, pp. 1-10, 2021. 